\title{
Applications of the New Compound Riccati Equations Rational Expansion Method and Fan's Subequation Method for the Davey-Stewartson Equations
}

\author{
Hassan Zedan ${ }^{1,2}$ \\ ${ }^{1}$ Mathematics Department, Faculty of Science, Kafr El-Sheikh University, Kafr El-Sheikh 33516, Egypt \\ ${ }^{2}$ Mathematics Department, Faculty of Science, King Abdul Aziz University, P.O. Box 80203, \\ Jeddah 21589, Saudi Arabia \\ Correspondence should be addressed to Hassan Zedan, hassanzedan2003@yahoo.com
}

Received 13 January 2010; Accepted 26 March 2010

Academic Editor: Vicentiu D. Radulescu

Copyright (C) 2010 Hassan Zedan. This is an open access article distributed under the Creative Commons Attribution License, which permits unrestricted use, distribution, and reproduction in any medium, provided the original work is properly cited.

\begin{abstract}
We used what we called extended Fan's sub-equation method and a new compound Riccati equations rational expansion method to construct the exact travelling wave solutions of the Davey-Stewartson (DS) equations. The basic idea of the proposed extended Fan's subequation method is to take fulls advantage of the general elliptic equations, involving five parameters, which have many new solutions and whose degeneracies lead to special subequations involving three parameters like Riccati equation, first-kind elliptic equation, auxiliary ordinary equation and generalized Riccati equation. Many new exact solutions of the Davey-Stewartson (DS) equations including more general soliton solutions, triangular solutions, and double-periodic solutions are constructed by symbolic computation.
\end{abstract}

\section{Introduction}

The investigation of the exact travelling wave solutions for nonlinear partial differential equations plays an important role in the study of nonlinear physical phenomena. These exact solutions when they exist can help the physicists to well understand the mechanism of the complicated physical phenomena and dynamically processes modeled by these NLPDEs. In recent years, large amounts of effort have been directed towards finding exact solutions. Many powerful method have been proposed, such as Darboux transformation [1], Hirota bilinear method [2], Lie group method [3], homogeneous balance method [4], tanh method. In this paper, we construct the exact travelling wave solutions for the Davey-Stewartson (DS) 
equations for the function $q=q(x, y, t)$ which are given by [5]

$$
\begin{aligned}
& i q_{t}+\frac{1}{2} \sigma^{2}\left(q_{x x}+\sigma^{2} q_{y y}\right)+\lambda|q|^{2} q-\phi_{x} q=0 \\
& \phi_{x x}-\sigma^{2} \phi_{y y}-2 \lambda\left(|q|^{2}\right)_{x}=0, \quad \lambda \pm 1, \sigma^{2} \pm 1.1 .
\end{aligned}
$$

The case $\sigma=1$ is called the DSI equation, while $\sigma=i$ is the DSII equation. The parameter $\lambda$ characterizes the focusing or defocusing case. The Davey-Stewartson I and II are two wellknown examples of integrable equations in two-dimensional space, which arise as higherdimensional generalizations of the nonlinear shrodinger (NLS) equation, from the point of physical view as well as from the study in [6]. Indeed, they appear in many applications, for example, in the description of gravity-capillarity surface wave packets and in the limit of the shallow water.

Davey and Stewartson first derived their model in the context of water waves, just purely physical considerations. In the context, $q(x, y, t)$ is the amplitude of a surface wave packet, while $\phi(x, y)$ is the velocity potential of the mean flow interacting with the surface wave [6].

The extended tanh-function method, the modified extended tanh-function method, and the F-expansion method belong to a class of methods called subequation methods for which they appear some basic relationships among the complicated NLPDEs under study and some simple solvable nonlinear ordinary equations. Thus by these subequation methods we seek for the solutions of the nonlinear partial differential equations in consideration as a polynomial in one variable satisfying equations (named subequation), for example, Riccati equation $\varphi^{\prime}=A+\varphi^{2}$, auxiliary ordinary equation $\varphi^{\prime 2}=B \varphi^{2}+C \varphi^{3}+D \varphi^{4}$ [7], first kind elliptic equation $\varphi^{\prime 2}=A+B \varphi^{2}+D \varphi^{4}$, generalized Riccati equation $\varphi^{\prime}=r+p \varphi+q \varphi^{2}$ [8], and so on. Fan [9] developed a new algebraic method, belonging to the class of subequation methods, to seek for more new solutions of nonlinear partial differential equations that can be expressed as a polynomial in an elementary function which satisfies a more general subequation than other subequations like Riccati equation, first-kind elliptic equation, and generalized Riccati equation. Recently Yomba [10] and Soliman and Abdou [11] extended Fan's method to show that the general elliptic equation can be degenerated in some special conditions to Riccati equation, first-kind elliptic equation, and generalized Riccati equation. We will consider a general elliptic equation in the formal will through

$$
\left(\frac{d \varphi(\xi)}{a \xi}\right)^{2}=c_{0}+c_{1} \varphi(\xi)+c_{2} \varphi^{2}(\xi)+c_{3} \varphi^{3}(\xi)+c_{4} \varphi^{4}(\xi)
$$

In addition, we apply a new compound Riccati equations rational expansion method [12] to the Davey-Stewartson (DS) equations and construct new complexion solutions. The rest of this paper is organized as follows. In Section 2, we simply provide the mathematical framework of Fan's subequation method. In Section 3, we apply the new presented method to the Davey-Stewartson (DS) equations. In Section 4, we briefly describe the new CRERE method. In Section 5, we obtain new complexion solutions of the Davey-Stewartson (DS) equations. In Section 6 and finally, some conclusions are given. 


\section{The Extended Fan Subequation Method}

In the following we shall outline the main steps of our method.

For given nonlinear partial differential equations with independent variables $x=\left(x_{0}=\right.$ $\left.t, x_{1}, x_{2}, \ldots, x_{l}\right)$ and dependent variable $u$,

$$
F\left(u, u_{t}, u_{x_{i}}, u_{x_{i} x_{j}}, \ldots\right)=0
$$

where $F$ is in general a polynomial function of its argument, and the subscripts denote the partial derivatives. We first consider its travelling wave solutions

$$
u=u(\xi), \quad \xi=\sum_{i=0}^{l} k_{i} x_{i}
$$

where $k_{i}(i=0, \ldots, l)$ are all arbitrary constants. Substituting (2.2) into (2.1), we get

$$
F\left(u, u^{\prime}, u^{\prime \prime}, \ldots\right)=0
$$

Then $u(\xi)$ is expanded into a polynomial in $\varphi(\xi)$ as

$$
u(\xi)=\sum_{j=0}^{n} a j \varphi^{j}(\xi)
$$

where $a_{j}$ are constants to be determined later and $\varphi(\xi)$ satisfies (1.2). In order to determine $u(\xi)$ explicitly, one may take the following steps.

Step 1. Determine $n$ by balancing the linear term of the highest order with the nonlinear term in (2.3).

Step 2. Substituting (2.4) with (1.2) into (2.3) and collecting all coefficients of $\varphi^{k}\left(\sqrt{\left(\sum_{p=0}^{4} c_{p} \varphi^{p}\right)^{v}}\right)(v=0,1 ; k=0,1,2, \ldots)$, then, setting these coefficients, to zero we get a set of algebraic equations with respect to $a_{j}(j=0,1, \ldots, n)$ and $k_{i}$.

Step 3. Solve the system of algebraic equations to obtain $a_{j}$ and $k_{j}$. Inserting these results into (2.4), we thus obtained the general form of travelling wave solutions.

Step 4. By using the results obtained in the above steps, we can derive a series of fundamental solutions to (1.2) depending on the different values chosen for $c_{0}, c_{1}, c_{2}, c_{3}$, and $c_{4}[7,8,10]$. The superscripts $I, I I, I I I, I V$, and $V$ determine the group of the solution while the subscript $l$ determines the rank of the solution. Those solutions are listed as follows. 
Case 1. In some special cases, when $c_{0} \neq 0, c_{1} \neq 0, c_{2} \neq 0, c_{3} \neq 0$, and $c_{4} \neq 0$, there may exist three parameters $r, p$, and $q$ such that

$$
\left(\frac{d \varphi(\xi)}{a \xi}\right)^{2}=c_{0}+c_{1} \varphi(\xi)+c_{2} \varphi^{2}(\xi)+c_{3} \varphi^{3}(\xi)+c_{4} \varphi^{4}(\xi)=\left(r+p \varphi(\xi)+q \varphi^{2}(\xi)\right)^{2}
$$

Equation (2.5) is satisfied only if the following relations hold:

$$
c_{0}=r^{2}, \quad c_{1}=2 r p, \quad c_{2}=2 r q+p^{2}, \quad c_{3}=2 p q, \quad c_{4}=q^{2} .
$$

For example, if the conditions (1.2)-(2.5) are satisfied, the following solutions are obtained [8].

Type 1. When $p^{2}-4 q r>0$ and $p q \neq 0(q r \neq 0)$,

$$
\begin{aligned}
& \varphi_{1}^{I}=-\frac{1}{2 q}\left[p+\sqrt{p^{2}-4 q r} \tanh \left(\frac{\sqrt{p^{2}-4 q r}}{2} \xi\right)\right] \\
& \varphi_{2}^{I}=-\frac{1}{2 q}\left[p+\sqrt{p^{2}-4 q r} \operatorname{coth}\left(\frac{\sqrt{p^{2}-4 q r}}{2} \xi\right)\right] \\
& \varphi_{3}^{I}=-\frac{1}{2 q}\left[p+\sqrt{p^{2}-4 q r} \tanh \left(\sqrt{p^{2}-4 q r} \xi\right) \pm i \operatorname{sech}\left(\sqrt{p^{2}-4 q r \xi}\right)\right] \\
& \varphi_{4}^{I}=-\frac{1}{2 q}\left[p+\sqrt{p^{2}-4 q r} \operatorname{coth}\left(\sqrt{p^{2}-4 q r} \xi\right) \pm \operatorname{csch}\left(\sqrt{p^{2}-4 q r \xi}\right)\right] \\
& \varphi_{5}^{I}=-\frac{1}{4 q}\left[2 p+\sqrt{p^{2}-4 q r} \tanh \left(\frac{\sqrt{p^{2}-4 q r}}{4} \xi\right) \pm \operatorname{coth}\left(\frac{\sqrt{p^{2}-4 q r}}{4} \xi\right)\right] \\
& \varphi_{6}^{I}=\frac{1}{2 q}\left[-p+\frac{\sqrt{\left(A^{2}+B^{2}\right)\left(p^{2}-4 q r\right)}-A \sqrt{p^{2}-4 q r} \cosh \left(\sqrt{p^{2}-4 q r} \xi\right)}{A \sinh \left(\sqrt{p^{2}-4 q r} \xi\right)+B}\right], \\
& \varphi_{7}^{I}=\frac{1}{2 q}\left[-p-\frac{\sqrt{\left(B^{2}-A^{2}\right)\left(p^{2}-4 q r\right)}+A \sqrt{p^{2}-4 q r} \sinh \left(\sqrt{p^{2}-4 q r} \xi\right)}{A \cosh \left(\sqrt{p^{2}-4 q r} \xi\right)+B}\right],
\end{aligned}
$$


where $A, B$ are two nonzero real constants and satisfy $B^{2}-A^{2}>0$;

$$
\begin{gathered}
\varphi_{8}^{I}=\frac{2 r \cosh \left(\left(\sqrt{p^{2}-4 q r} / 2\right) \xi\right)}{\sqrt{p^{2}-4 q r} \sinh \left(\left(\sqrt{p^{2}-4 q r} / 2\right) \xi\right)-p \cosh \left(\left(\sqrt{p^{2}-4 q r} / 2\right) \xi\right)}, \\
\varphi_{9}^{I}=\frac{-2 r \sinh \left(\left(\sqrt{p^{2}-4 q r} / 2\right) \xi\right)}{p \sinh \left(\left(\sqrt{p^{2}-4 q r} / 2\right) \xi\right)-\sqrt{p^{2}-4 q r} \cosh \left(\left(\sqrt{p^{2}-4 q r} / 2\right) \xi\right)}, \\
\varphi_{10}^{I}=\frac{2 r \cosh \left(\sqrt{p^{2}-4 q r} \xi\right)}{\sqrt{p^{2}-4 q r} \sinh \left(\sqrt{p^{2}-4 q r} \xi\right)-p \cosh \left(\sqrt{p^{2}-4 q r} \xi\right)+i \sqrt{p^{2}-4 q r}}, \\
\varphi_{11}^{I}=\frac{2 r \sinh \left(\sqrt{p^{2}-4 q r \xi}\right)}{-p \sinh \left(\sqrt{p^{2}-4 q r} \xi\right)+\sqrt{p^{2}-4 q r} \cosh \left(\sqrt{p^{2}-4 q r} \xi\right) \pm \sqrt{p^{2}-4 q r}}, \\
\varphi_{12}^{I}=\frac{4 r \cosh \left(\left(\sqrt{p^{2}-4 q r} / 4\right) \xi\right) \sinh \left(\left(\sqrt{p^{2}-4 q r} / 4\right) \xi\right)}{-2 p \cosh \left(\left(\sqrt{p^{2}-4 q r} / 4\right) \xi\right) \sinh \left(\left(\sqrt{p^{2}-4 q r} / 4\right) \xi\right)+\mathfrak{A}},
\end{gathered}
$$

where $\mathfrak{A}$ denotes $2 \sqrt{p^{2}-4 q r} \cosh ^{2}\left(\left(\sqrt{p^{2}-4 q r} / 4\right) \xi\right)-\sqrt{p^{2}-4 q r}$.

Type 2. When $p^{2}-4 q r<0$ and $p q \neq 0(q r \neq 0)$

$$
\begin{gathered}
\varphi_{13}^{I}=\frac{1}{2 q}\left[-p+\sqrt{4 q r-p^{2}}\left(\frac{\sqrt{4 q r-p^{2}}}{2} \xi\right)\right] \\
\varphi_{14}^{I}=-\frac{1}{2 q}\left[p+\sqrt{4 q r-p^{2}} \cot \left(\frac{\sqrt{4 q r-p^{2}}}{2} \xi\right)\right] \\
\varphi_{15}^{I}=\frac{1}{2 q}\left[p+\sqrt{4 q r-p^{2}} \tan \left(\sqrt{4 q r-p^{2}} \xi\right) \pm I \sec \left(\sqrt{4 q r-p^{2}} \xi\right)\right] \\
\varphi_{16}^{I}=-\frac{1}{2 q}\left[p+\sqrt{4 q r-p^{2}} \cot \left(\sqrt{4 q r-p^{2}} \xi\right) \pm \csc \left(\sqrt{4 q r-p^{2}} \xi\right)\right], \\
\varphi_{17}^{I}=\frac{1}{4 q}\left[-2 p+\sqrt{4 q r-p^{2}} \tan \left(\frac{\sqrt{4 q r-p^{2}}}{4} \xi\right)-\cot \left(\frac{\sqrt{4 q r-p^{2}}}{4} \xi\right),\right.
\end{gathered}
$$




$$
\begin{aligned}
& \varphi_{18}^{I}=\frac{1}{2 q}\left[-p+\frac{ \pm \sqrt{\left(A^{2}-B^{2}\right)\left(4 q r-p^{2}\right)}-A \sqrt{4 q r-p^{2}} \cos \left(\sqrt{4 q r-p^{2}} \xi\right)}{A \sin \left(\sqrt{4 q r-p^{2}} \xi\right)+B}\right], \\
& \varphi_{19}^{I}=\frac{1}{2 q}\left[-p-\frac{ \pm \sqrt{\left(A^{2}-B^{2}\right)\left(4 q r-p^{2}\right)}-A \sqrt{4 q r-p^{2}} \sin \left(\sqrt{4 q r-p^{2}} \xi\right)}{A \cos \left(\sqrt{4 q r-p^{2}} \xi\right)+B}\right],
\end{aligned}
$$

where $A, B$ are two nonzero real constants and satisfy $A^{2}-B^{2}>0$;

$$
\begin{gathered}
\varphi_{20}^{I}=-\frac{2 r \cos \left(\left(\sqrt{4 q r-p^{2}} / 2\right) \xi\right)}{\sqrt{4 q r-p^{2}} \sin \left(\left(\sqrt{4 q r-p^{2}} / 2\right) \xi\right)+p \cos \left(\left(\sqrt{4 q r-p^{2}} / 2\right) \xi\right)}, \\
\varphi_{21}^{I}=\frac{2 r \sin \left(\left(\sqrt{4 q r-p^{2}} / 2\right) \xi\right)}{-p \sin \left(\left(\sqrt{4 q r-p^{2}} / 2\right) \xi\right)+\sqrt{4 q r-p^{2}} \cos \left(\left(\sqrt{4 q r-p^{2}} / 2\right) \xi\right)}, \\
\varphi_{22}^{I}=-\frac{2 r \cos \left(\sqrt{4 q r-p^{2}} \xi\right)}{\sqrt{4 q r-p^{2}} \sin \left(\sqrt{4 q r-p^{2}} \xi\right)+p \cos \left(\sqrt{4 q r-p^{2}} \xi\right) \pm \sqrt{4 q r-p^{2}}}, \\
\varphi_{23}^{I}=\frac{2 r \sin \left(\sqrt{4 q r-p^{2}} \xi\right)}{-p \sin \left(\sqrt{4 q r-p^{2}} \xi\right)+\sqrt{4 q r}-p^{2} \cos \left(\sqrt{4 q r-p^{2}} \xi\right) \pm \sqrt{4 q r-p^{2}}}, \\
\varphi_{24}^{I}=\frac{4 r \cos \left(\left(\sqrt{4 q r-p^{2}} / 4\right) \xi\right) \sin \left(\left(\sqrt{4 q r-p^{2}} / 4\right) \xi\right)}{-2 p \cos \left(\left(\sqrt{4 q r-p^{2}} / 4\right) \xi\right) \sin \left(\left(\sqrt{4 q r-p^{2}} / 4\right) \xi\right)+\mathfrak{B}^{\prime}},
\end{gathered}
$$

where $\mathfrak{B}$ denotes $2 \sqrt{4 q r-p^{2}} \cosh ^{2}\left(\left(\sqrt{4 q r-p^{2}} / 4\right) \xi\right)-\sqrt{4 q r-p^{2}}$.

Case 2. Case 1 includes another special case when $c_{2}=0$ and $c_{1} \neq 0, c_{0} \neq 0, c_{3} \neq 0$, and $c_{4} \neq 0$. There may exist three parameters $r, p$, and $q$ such that

$$
\left(\frac{d \varphi(\xi)}{a \xi}\right)^{2}=c_{0}+c_{1} \varphi(\xi)+c_{3} \varphi^{3}(\xi)+c_{4} \varphi^{4}(\xi)=\left(r+p \varphi(\xi)+q \varphi^{2}(\xi)\right)^{2}
$$

Equation (2.2) requires for its existence the following relations:

$$
c_{0}=r^{2}, \quad c_{1}=2 r p, \quad c_{3}=2 p q, \quad c_{4}=q^{2} .
$$


The following constraint should exist between $r, p$, and $q$ parameters:

$$
p^{2}=-2 r q, \quad r q<0
$$

For example, if the conditions (1.2), (2.5), (2.12), and (2.13) are satisfied, the following solutions are obtained.

Type 1 . When $q r<0$ and $(q r \neq 0)$,

$$
\begin{gathered}
\varphi_{1}^{I I}=-\frac{1}{2 q}\left[ \pm \sqrt{-2 q r}+\sqrt{-6 q r} \tanh \left(\frac{\sqrt{-6 q r}}{2} \xi\right)\right], \\
\varphi_{2}^{I I}=-\frac{1}{2 q}\left[ \pm \sqrt{-2 q r}+\sqrt{-6 q r} \operatorname{coth}\left(\frac{\sqrt{-6 q r}}{2} \xi\right)\right], \\
\varphi_{3}^{I I}=-\frac{1}{2 q}[ \pm \sqrt{-2 q r}+\sqrt{-6 q r} \tanh (\sqrt{-6 q r} \xi) \pm i \operatorname{sech}(\sqrt{-6 q r \xi})], \\
\varphi_{4}^{I I}=-\frac{1}{2 q}[ \pm \sqrt{-2 q r}+\sqrt{-6 q r} \operatorname{coth}(\sqrt{-6 q r \xi}) \pm \operatorname{csch}(\sqrt{-6 q r \xi})], \\
\varphi_{5}^{I I}=-\frac{1}{4 q}\left[ \pm 2 \sqrt{-2 q r}+\sqrt{-6 q r} \tanh \left(\frac{\sqrt{-6 q r}}{4} \xi\right)+\cot h\left(\frac{\sqrt{-6 q r}}{4} \xi\right)\right], \\
\varphi_{6}^{I I}=\frac{1}{2 q}\left[\mp \sqrt{-2 q r}+\frac{\sqrt{\left(A^{2}+B^{2}\right)(-6 q r)}-A \sqrt{-6 q r} \cosh (\sqrt{-6 q r} \xi)}{A \sinh (\sqrt{-6 q r} \xi)+B}\right], \\
\varphi_{7}^{I I}=\frac{1}{2 q}\left[\mp \sqrt{-2 q r}-\frac{\sqrt{\left(B^{2}-A^{2}\right)(-6 q r)}+A \sqrt{-6 q r} \sinh (\sqrt{-6 q r} \xi)}{A \cosh (\sqrt{-6 q r} \xi)+B}\right],
\end{gathered}
$$

where $A, B$ are two nonzero real constants and satisfy $B^{2}-A^{2}>0$;

$$
\begin{gathered}
\varphi_{8}^{I I}=\frac{2 r \cosh ((\sqrt{-6 q r} / 2) \xi)}{\sqrt{-6 q r} \sinh ((\sqrt{-6 q r} / 2) \xi) \mp \sqrt{-2 q r} \cosh ((\sqrt{-6 q r} / 2) \xi)}, \\
\varphi_{9}^{I I}=\frac{-2 r \sinh ((\sqrt{-6 q r} / 2) \xi)}{ \pm \sqrt{-2 q r} \sinh ((\sqrt{-6 q r} / 2) \xi)-\sqrt{-6 q r} \cosh ((\sqrt{-6 q r} / 2) \xi)}, \\
\varphi_{10}^{I I}=\frac{2 r \cosh (\sqrt{-6 q r} \xi)}{\sqrt{-6 q r} \sinh (\sqrt{-6 q r} \xi) \mp \sqrt{-2 q r} \cosh (\sqrt{-6 q r} \xi)+i \sqrt{-6 q r}}, \\
\varphi_{11}^{I I}=\frac{2 r \sinh (\sqrt{-6 q r} \xi)}{\mp \sqrt{-2 q r} \sinh (\sqrt{-6 q r} \xi)+\sqrt{-6 q r} \cosh (\sqrt{-6 q r} \xi) \pm \sqrt{-6 q r}}, \\
\varphi_{12}^{I I}=\frac{4 r \cosh ((\sqrt{-6 q r} / 4) \xi) \sinh ((\sqrt{-6 q r} / 4) \xi)}{\mp 2 \sqrt{-2 q r} \cosh ((\sqrt{-6 q r} / 4) \xi) \sinh ((\sqrt{-6 q r} / 4) \xi)+\mathfrak{C}^{\prime}},
\end{gathered}
$$


where $\mathfrak{C}$ denotes $2 \sqrt{-6 q r} \cosh ^{2}((\sqrt{-6 q r} / 4) \xi)-\sqrt{-6 q r}$. Thus for (2.5), (2.11), the general elliptic equation is reduced to the generalized Riccati Equation [8].

Case 3. When $c_{0}=c_{1}=0$, the general elliptic equation is reduced to the auxiliary ordinary equation [7]

$$
\left(\frac{d \varphi(\xi)}{a \xi}\right)^{2}=c_{2} \varphi^{2}(\xi)+c_{3} \varphi^{3}(\xi)+c_{4} \varphi^{4}(\xi)
$$

For example, if the condition (2.16) is satisfied, the following solutions are obtained.

Type 1. If $c_{2}=1, c_{3}=-2 c / a$, and $c_{4}=\left(c^{2}-b^{2}\right) / a^{2},(2.16)$ has the solution

$$
\varphi_{1}^{I I I}=\frac{a \sec h \xi}{b+c \sec h \xi} .
$$

Type 2. If $c_{2}=1, c_{3}=-2 c / a$, and $c_{4}=\left(c^{2}+b^{2}\right) / a^{2},(2.16)$ has the solution

$$
\varphi_{2}^{I I I}=\frac{a \operatorname{csch} \xi}{b+c \operatorname{csch} \xi}
$$

Type 3. If $c_{2}=4, c_{3}=-(4(2 b+d)) / a$, and $c_{4}=\left(c^{2}+4 b^{2}+4 b d\right) / a^{2},(2.16)$ has the solution

$$
\varphi_{3}^{I I I}=\frac{a \sec h^{2} \xi}{b \sec h^{2} \xi+c \tanh \xi+d} .
$$

Type 4. If $c_{2}=4, c_{3}=(4(d-2 b)) / a$, and $c_{4}=\left(c^{2}+4 b^{2}-4 b d\right) / a^{2},(2.16)$ has the solution

$$
\varphi_{4}^{I I I}=\frac{a \operatorname{csch} h^{2} \xi}{b \operatorname{csch} h^{2}+c \operatorname{coth} \xi+d} .
$$

Type 5. If $c_{2}=a^{2}, c_{3}=2 a b$, and $c_{4}=b^{2},(2.16)$ has the solution

$$
\begin{aligned}
\varphi_{5}^{I I I} & =\frac{-a c}{b(c+\cosh (a \xi)-\sinh (a \xi))} \\
\varphi_{6}^{I I I} & =-\frac{a(\cosh (a \xi)+\sinh (a \xi))}{b(c+\cosh (a \xi)+\sinh (a \xi))} .
\end{aligned}
$$


Type 6. If $c_{2}=-1, c_{3}=2 c / a$, and $c_{4}=-\left(c^{2}-b^{2}\right) / a^{2}$, (2.16) has the solution

$$
\begin{aligned}
& \varphi_{7}^{I I I}=\frac{a \sec \xi}{b+c \sec \xi}, \\
& \varphi_{8}^{I I I}=\frac{a \csc \xi}{b+c \csc \xi} .
\end{aligned}
$$

Type 7. If $c_{2}=-4, c_{3}=4(2 b+d) / a$, and $c_{4}=-\left(-c^{2}+4 b^{2}+4 b d\right) / a^{2},(2.16)$ has the solution

$$
\begin{aligned}
\varphi_{9}^{I I I} & =\frac{a \sec ^{2} \xi}{b \sec ^{2} \xi+c \tan \xi+d} \\
\varphi_{10}^{I I I} & =\frac{a \csc ^{2} \xi}{b \csc ^{2} \xi+c \cot \xi+d}
\end{aligned}
$$

where $a, b, c$, and $d$ are arbitrary constants.

Type 8. If $c_{2}>0$,

$$
\varphi_{11}^{I I I}=-\frac{c_{2} c_{3} \sec h^{2}\left(\left(\sqrt{c_{2}} / 2\right) \xi\right)}{c_{3}^{2}-c_{2} c_{4}\left(1-\tanh \left(\left(\sqrt{c_{2}} / 2\right) \xi\right)\right)^{2}} .
$$

When $c_{1}=c_{3}=0$, the general elliptic equation is reduced to the elliptic equation

$$
\left(\frac{d \varphi(\xi)}{a \xi}\right)^{2}=c_{0}+c_{2} \varphi^{2}(\xi)+c_{4} \varphi^{4}(\xi)
$$

Equation (2.25) includes the Riccati equation

$$
\left(\frac{d \varphi(\xi)}{a \xi}\right)^{2}=\left(A+\varphi^{2}(\xi)\right)^{2}
$$

where $c_{0}=A^{2}, c_{2}=2 A, c_{4}=1$, and solutions of (2.26) can be deduced from those of (2.25) in the specific case where the modulus $m$ of the Jacobi elliptic functions is drived to 1 and 0 .

Case 4. Assume that the conditions of verification of (2.26) are fulfilled, then the general solutions are just the single solution and the combined nondegenerative Jacobi elliptic functions. The relations between the values of $\left(c_{0}, c_{2}, c_{4}\right)$ and the corresponding Jacobi elliptic function solution of the NODE (2.25) are given in Table 1.

where the modulus $m$ of the Jacobi elliptic function satisfies $(0 \leq m \leq 1)$. Table 2).

The Jacobi elliptic function degenerates as hyperbolic functions when $m \rightarrow 1$ (see Table 3).

The Jacobi elliptic function degenerates as hyperbolic functions when $m \rightarrow 0$ (see 
Table 1

\begin{tabular}{|c|c|c|c|}
\hline$\underline{\varphi}$ & $c_{0}$ & $c_{2}$ & $c_{4}$ \\
\hline $\operatorname{sn} \xi ; \mathrm{cd} \xi=\mathrm{cn} \xi / \mathrm{dn} \xi$ & 1 & $-\left(m^{2}+1\right)$ & $m^{2}$ \\
\hline $\mathrm{cn} \xi$ & $1-m^{2}$ & $2 m^{2}-1$ & $-m^{2}$ \\
\hline $\operatorname{dn} \xi$ & $m^{2}-1$ & $2-m^{2}$ & -1 \\
\hline $\mathrm{ns} \xi=1 / \mathrm{sn} \xi ; \mathrm{dc} \xi=\mathrm{dn} \xi / \mathrm{cn} \xi$ & $m^{2}$ & $-\left(m^{2}+1\right)$ & 1 \\
\hline $\mathrm{nc} \xi=1 / \mathrm{cn} \xi$ & $-m^{2}$ & $2 m^{2}-1$ & $1-m^{2}$ \\
\hline nd $\xi=1 / \mathrm{dn} \xi$ & -1 & $2-m^{2}$ & $m^{2}-1$ \\
\hline $\operatorname{cs} \xi=\mathrm{cn} \xi / \mathrm{sn} \xi$ & $1-m^{2}$ & $2-m^{2}$ & 1 \\
\hline $\mathrm{sc} \xi=1 / \mathrm{cs}$ & 1 & $2-m^{2}$ & $1-m^{2}$ \\
\hline $\operatorname{sd} \xi=\operatorname{sn} \xi / \mathrm{dn} \xi$ & 1 & $2 m^{2}-1$ & $m^{2}\left(m^{2}-1\right)$ \\
\hline $\mathrm{ds} \xi=1 / \mathrm{sd} \xi$ & $m^{2}\left(m^{2}-1\right)$ & $2 m^{2}-1$ & 1 \\
\hline$m \mathrm{cn} \xi \pm \mathrm{dn} \xi$ & $-\left(1-m^{2}\right) 2 / 4$ & $\left(1+m^{2}\right) / 2$ & $-1 / 4$ \\
\hline $\mathrm{ns} \xi \pm \mathrm{cs} \xi$ & $1 / 4$ & $\left(1-2 m^{2}\right) / 2$ & $1 / 4$ \\
\hline $\mathrm{nc} \xi \pm \mathrm{sc} \xi$ & $\left(1-m^{2}\right) / 4$ & $\left(1+m^{2}\right) / 2$ & $\left(1-m^{2}\right) / 4$ \\
\hline $\mathrm{ns} \xi \pm \mathrm{ds} \xi$ & $m^{4} / 4$ & $\left(m^{2}-2\right) / 2$ & $1 / 4$ \\
\hline $\operatorname{sn} \xi \pm i \mathrm{cn} \xi ; \mathrm{dn} \xi /\left(\sqrt{1-m^{2}} \operatorname{sn} \xi \pm \mathrm{cn} \xi\right)$ & $m^{2} / 4$ & $\left(m^{2}-2\right) / 2$ & $m^{2} / 4$ \\
\hline$m \operatorname{sn} \xi \pm i \mathrm{dn} \xi ; \mathrm{sn} \xi /(1 \pm \mathrm{cn} \xi)$ & $1 / 4$ & $\left(1-2 m^{2}\right) / 2$ & $1 / 4$ \\
\hline $\operatorname{sn} \xi /(1 \pm \operatorname{dn} \xi)$ & $1 / 4$ & $\left(m^{2}-2\right) / 2$ & $m^{2} / 4$ \\
\hline $\mathrm{dn} \xi /(1 \pm m \operatorname{sn} \xi)$ & $\left(m^{2}-1\right) / 4$ & $\left(m^{2}+1\right) / 2$ & $\left(m^{2}-1\right) / 4$ \\
\hline $\mathrm{cn} \xi /(1 \pm \operatorname{sn} \xi)$ & $\left(1-m^{2}\right) / 4$ & $\left(m^{2}+1\right) / 2$ & $\left(1-m^{2}\right) / 4$ \\
\hline $\operatorname{sn} \xi /(\mathrm{dn} \xi \pm \mathrm{cn} \xi)$ & $1 / 4$ & $\left(m^{2}+1\right) / 2$ & $\left(1-m^{2}\right) / 4$ \\
\hline $\mathrm{cn} \xi /\left(\sqrt{1-m^{2}} \pm \mathrm{dn} \xi\right)$ & $1 / 4$ & $\left(m^{2}-2\right) / 2$ & $m^{4} / 4$ \\
\hline
\end{tabular}

Table 2

\begin{tabular}{lccccc}
\hline $\operatorname{sn} \xi$ & $\mathrm{cn} \xi$ & $\mathrm{dn} \xi$ & $\mathrm{sc} \xi$ & $\mathrm{sd} \xi$ & $\mathrm{cd} \xi$ \\
$\tanh \xi$ & $\operatorname{sech} \xi$ & $\operatorname{sech} \xi$ & $\sinh \xi$ & $\sinh \xi$ & 1 \\
$\operatorname{ns} \xi$ & $\operatorname{nc} \xi$ & $\operatorname{cs} \xi$ & $\mathrm{ds} \xi$ & $\mathrm{dc} \xi$ \\
$\operatorname{coth} \xi$ & $\cosh \xi$ & $\cosh \xi$ & $\operatorname{csch} \xi$ & $\operatorname{csch} \xi$ & 1 \\
\hline
\end{tabular}

Table 3

\begin{tabular}{lccccc}
\hline $\operatorname{sn} \xi$ & $\mathrm{cn} \xi$ & $\mathrm{dn} \xi$ & $\mathrm{sc} \xi$ & $\mathrm{sd} \xi$ & $\mathrm{cd} \xi$ \\
$\sin \xi$ & 1 & $\tan \xi$ & $\sin \xi$ & $\cos \xi$ \\
$\mathrm{ns} \xi$ & $\cos \xi$ & $\mathrm{nd} \xi$ & $\mathrm{cs} \xi$ & $\mathrm{ds} \xi$ & $\mathrm{dc} \xi$ \\
$\csc \xi$ & $\mathrm{nc} \xi$ & 1 & $\cot \xi$ & $\csc \xi$ & $\sec \xi$ \\
\hline
\end{tabular}

Case 5. When $c_{4}=c_{2}=0$, the general elliptic equation is reduced to the following:

$$
\left(\frac{d \varphi(\xi)}{a \xi}\right)^{2}=c_{0}+c_{1} \varphi(\xi)+c_{3} \varphi^{3}(\xi)
$$


For example, if the condition (2.27) holds, the solution is of Weierstrass elliptic doubly periodic type

$$
\varphi_{1}^{V}=\vartheta\left(\frac{\sqrt{h_{3}}}{2} \xi, g_{2}, g_{3}\right), \quad h_{3}>0
$$

where $g_{2}=-4 c_{1} / c_{3} ; g_{3}=-4 c_{0} / c_{3}$.

Case 6. When $c_{0}=c_{1}=c_{2}=c_{3}=0$, and $c_{4}=1$, and $c_{3} \neq 0$, the general elliptic equation admits solution

$$
\varphi(\xi)=\frac{ \pm 1}{\xi+C}
$$

Case 7. when $c_{0}=c_{1}=c_{4}=0$ and $c_{2}=1$, the general elliptic equation have solution

$$
\varphi(\xi)=\frac{2}{c_{3}} \csc h^{2}(\xi) \pm \frac{2}{c_{3}} \csc h(\xi) \operatorname{coth}(\xi)
$$

\section{Exact Solutions of the Davey-Stewartson (DS) Equations}

Now, we will construct the exact solutions to (DS) equations (1.1). Let us assume the travelling wave solutions of (1.1) in the form

$$
\begin{gathered}
q(x, y, t)=e^{i \theta} u(\xi), \quad \phi(x, y, t)=v(\xi), \\
\xi=\alpha x+\beta y+\gamma t, \quad \theta=k x+c y+r t,
\end{gathered}
$$

where $u(\xi), v(\xi)$ are real functions, and the constants $\alpha, \beta, \gamma, k, c, t$ are real which can be determined later. Substituting (3.1) into (1.1), we find that $\gamma=-\sigma^{2}(\alpha k+\beta c)$, and $u, v$ satisfy the following coupled ordinary differential system:

$$
\begin{gathered}
\sigma^{2}\left(\alpha^{2}+\sigma^{2} \beta^{2}\right) u^{\prime \prime}-2 \alpha u v^{\prime}+2 \lambda u^{3}-\left(2 r+\sigma^{2} k^{2}+\sigma^{4} c^{2}\right) u=0, \\
\left(\alpha^{2}-\sigma^{2} \beta^{2}\right) v^{\prime \prime}-2 \lambda \alpha\left(u^{2}\right)^{\prime}=0,
\end{gathered}
$$

where "the prime" denotes to $d / d \xi$.

Integrating (3.3) w.r.t. $\xi$ and solving for $v^{\prime}(\xi)$, we get

$$
v^{\prime}(\xi)=\frac{2 \lambda \alpha}{\alpha^{2}-\sigma^{2} \beta^{2}} u^{2}+C_{1}
$$

where $C_{1}$ is an integration constant. Substituting (3.4) into (3.2), we get

$$
k_{1} u^{\prime \prime}+k_{2} u^{3}+k_{4} u=0 \text {, }
$$


where

$$
\begin{gathered}
k_{1}=\sigma^{2}\left(\alpha^{2}+\sigma^{2} \beta^{2}\right), \\
k_{2}=2 \lambda-\frac{4 \lambda \alpha^{2}}{\left(\alpha^{2}-\sigma^{2} \beta^{2}\right)} \\
k_{3}=0, \\
k_{4}=-\left(2 \alpha c_{1}+2 r+\sigma^{2} k^{2}+\sigma^{2} c^{2}\right) .
\end{gathered}
$$

Balancing the highest-order derivative term $\left(u^{\prime \prime}\right)$ with nonlinear term $\left(u^{3}\right)$ in (3.5) gives leading $n=1$. We thus suppose that (3.5) has the following formal solutions:

$$
u(\xi)=a_{0}+a_{1} \varphi(\xi)
$$

where $a_{0}, a_{1}$ are to be determined later; substituting (3.7) along with (1.2) into (3.5) yields a polynomial equation in $\varphi$. Setting to zero their coefficients yields the following set of algebraic equations:

$$
\begin{gathered}
\frac{1}{2} a_{1} c_{1} k_{1}+a_{0}^{3} k_{2}+a_{0} k_{4}=0 \\
a_{1} c_{2} k_{1}+3 a_{0}^{3} a_{1} k_{2}+a_{1} k_{4}=0 \\
\frac{3}{2} a_{1} c_{3} k_{1}+3 a_{0} a_{1}^{2} k_{2}=0 \\
2 a_{1} c_{4} k_{1}+a_{1}^{3} k_{2}=0
\end{gathered}
$$

Substituting (3.6) into (3.8) and solving with respect to $a_{0}, a_{1}, \alpha, \beta, k, c, r$, we obtain the following solutions:

$$
\begin{gathered}
a_{0}=\frac{1}{2} \sqrt{\frac{-c_{3}^{2} \beta^{2} \sigma^{4}}{2 \lambda c_{4}}}, \quad a_{1}=\sqrt{\frac{-2 c_{4} \beta^{2} \sigma^{4}}{\lambda}}, \\
\alpha=\mp \sqrt{-\beta^{2} \sigma^{2}}, \quad r=-\frac{1}{2} k^{2} \sigma^{2}-\frac{1}{2} c^{2} \sigma^{4} \pm C_{1} \sqrt{-\beta^{2} \sigma^{2}},
\end{gathered}
$$

where $\beta, k, c$ are arbitrary real constants, and $a_{0}, a_{1}, \alpha, r$ are real constants so we choose the case $\sigma=i$.

The exact travelling wave solutions of the DSII equations (1.1) are given by

$$
\begin{gathered}
q(x, y, t)=e^{i \theta}\left(a_{0}+a_{1} \varphi(\xi)\right), \\
\phi(x, y, t)=\int\left[r_{1}\left(a_{0}+a_{1} \varphi(\xi)\right)^{2}+C_{1}\right] d \xi, \quad r_{1}=\frac{2 \lambda \alpha}{\alpha^{2}+\beta^{2}}
\end{gathered}
$$


where

$$
\begin{gathered}
a_{0}=\frac{1}{2} \beta c_{3} \sqrt{\frac{-1}{2 \lambda c_{4}}}, \\
a_{1}=\beta \sqrt{\frac{-2 c_{4}}{\ell}}, \quad c_{4} \curlywedge<0 \quad \text { or } \quad c_{4}<0, \\
\xi=\beta[\mp x+y+(\mp k+c) t], \quad \theta=k x+c y+\left(\frac{1}{2} k^{2}-\frac{1}{2} c^{2} \pm \beta C_{1}\right),
\end{gathered}
$$

and $c_{i}(i=0,1,2,3,4)$ are arbitrary constants. We may have many kinds of solutions depending on the special values for $c_{i}$.

Case 1. If $c_{0}=r^{2}, c_{1}=2 r p, c_{2}=2 r q+p^{2}, c_{3}=2 p q$, and $c_{4}=q^{2}$, then $\varphi$ is one of the $24 \varphi_{l}^{I}(l=1,2, \ldots, 24)$ containing $22 \varphi_{l}^{I}$ that are real and $2 \varphi_{l}^{I}$ that are complex. For example, if we select $(=1,15)$, then one could write down explicitly the following soliton solutions:

$$
\begin{aligned}
& q_{1}(x, y, t)=e^{i \theta}\left[a_{0}-\frac{a_{1}}{2 q}\left(p+\beta_{0} \tanh \left(\frac{\beta_{0}}{2} \xi\right)\right)\right], \\
& \phi_{1}(x, y, t)=\frac{1}{4 q^{2}}\left[\left(4 C_{1} q^{2}+r_{1}\left(\left(a_{1} p-2 a_{0} q\right)^{2}+a_{1}^{2} \beta_{0}\right)\right) \xi\right. \\
& \left.+4 a_{1}\left(a_{1} p-2 a_{0} q\right) r_{1} \ln \left(\cosh \left(\frac{\beta_{0}}{2} \xi\right)\right)-2 a_{1}^{2} r_{1} \beta_{0} \tanh \left(\frac{\beta_{0}}{2} \xi\right)\right] \\
& q_{2}(x, y, t)=e^{i \theta}\left[a_{0}+\frac{a_{1}}{2 q}\left(-p+\beta_{1}\left(\tan \beta_{1} \xi \pm \sec \beta_{1} \xi\right)\right)\right], \\
& \phi_{2}(x, y, t)=C_{1} \xi+\frac{1}{4 q^{2}}\left[r_{1}\left(4 a_{0}^{2} q^{2}-4 a_{0} a_{1} p q-4 a_{1}^{2} q r\right) \xi\right. \\
& +4 a_{1} r_{1}\left(a_{1} p-2 a_{0} q\right) \ln \left(\cos \frac{\beta_{1}}{2} \xi \mp \sin \frac{\beta_{1}}{2} \xi\right) \\
& \left.+4 a_{1}^{2} r_{1} \beta_{1} \frac{\sin \left(\left(\beta_{1} / 2\right) \xi\right)}{\left(\cos \left(\left(\beta_{1} / 2\right) \xi\right) \mp \sin \left(\left(\beta_{1} / 2\right) \xi\right)\right)}\right] \text {, }
\end{aligned}
$$

where

$$
\begin{gathered}
\beta_{0}=\sqrt{p^{2}-4 p q}, \quad \beta_{1}=\sqrt{4 p q-p^{2}}, \\
a_{0}=p \beta \sqrt{\frac{-1}{2 \lambda}}, \quad a_{1}=q \beta \sqrt{\frac{-2}{\lambda}}, \quad \lambda<0, \\
\xi=\beta[\mp x+y+(\mp k+c) t], \quad \theta=k x+c y+\left(\frac{1}{2} k^{2}-\frac{1}{2} c^{2} \pm \beta C_{1}\right) .
\end{gathered}
$$


Case 2. If $c_{0}=r^{2}, c_{1}=2 r p, c_{2}=0, c_{3}=2 p q, c_{4}=q^{2}, p^{2}=-2 r q$, then $\varphi$ is one of the $12 \varphi_{l}^{I}(l=1,2, \ldots, 12)$ contain $10 \varphi_{l}^{I}$ are real and $2 \varphi_{l}^{I}$ are complex. For example, if we select $(l=1,4)$, then one may write down explicitly the following soliton solutions:

$$
\begin{aligned}
q_{3}(x, y, t)=e^{i \theta}[ & \left.a_{0}-\frac{a_{1}}{2 q}\left(\beta_{2}+\beta_{3} \tanh \left(\frac{\beta_{3}}{2} \xi\right)\right)\right], \\
\phi_{3}(x, y, t)=\frac{1}{4 q^{2}} & {\left[\left(4 C_{1} q^{2}+r_{1}\left(4 a_{0}^{2} q^{2}-4 a_{0} a_{1} q \beta_{2}-8 a_{1}^{2} q r\right)\right) \xi\right.} \\
& \left.+4 a_{1} r_{1}\left(-2 a_{0} q+a_{1} \beta_{2}\right) \ln \left(\cosh \left(\frac{\beta_{3}}{2} \xi\right)\right)-2 a_{1}^{2} r_{1} \beta_{3} \tanh \left(\frac{\beta_{3}}{2} \xi\right)\right] \\
q_{4}(x, y, t)=e^{i \theta}[ & \left.a_{0}-\frac{a_{1}}{2 q}\left(\beta_{2}+\beta_{3}\left(\operatorname{coth}\left(\frac{\beta_{3}}{2} \xi\right)+\operatorname{csch}\left(\frac{\beta_{3}}{2} \xi\right)\right)\right)\right], \\
\phi_{4}(x, y, t)=\frac{1}{4 q^{2}}\left[\left(4 C_{1} q^{2}+r_{1}\left(4 a_{0}^{2} q^{2}-4 a_{0} a_{1} q \beta_{2}-8 a_{1}^{2} q r\right)\right) \xi\right. & \left.+4 a_{1} r_{1}\left(-2 a_{0} q+a_{1} \beta_{2}\right) \ln \left(\sinh \left(\frac{\beta_{3}}{2} \xi\right)\right)-2 a_{1}^{2} r_{1} \beta_{3} \operatorname{coth}\left(\frac{\beta_{3}}{2} \xi\right)\right],
\end{aligned}
$$

where

$$
\begin{gathered}
\beta_{2}=\sqrt{-2 q r}, \quad \beta_{3}=\sqrt{-6 q r}, \\
a_{0}=p \beta \sqrt{\frac{-1}{2 \lambda},} \quad a_{1}=q \beta \sqrt{\frac{-2}{\lambda}}, \quad \lambda<0 \\
\xi=\beta[\mp x+y+(\mp k+c) t], \quad \theta=k x+c y+\left(\frac{1}{2} k^{2}-\frac{1}{2} c^{2} \pm \beta C_{1}\right) .
\end{gathered}
$$

Case 3. If $c_{0}=c_{1}=0$, and $c_{2}, c_{3}, c_{4}$ are arbitrary constants, then $\varphi$ is one of the $10 \varphi_{l}^{I}(l=$ $1,2, \ldots, 10)$ as follows.

Type 1. If $c_{2}=1, c_{3}=-2 c / a$, and $c_{4}=\left(c^{2}-b^{2}\right) / a^{2}$, then the travelling wave solutions are given as follows:

$$
\begin{aligned}
q_{5}(x, y, t)= & e^{i \theta}\left[a_{0}+\frac{a_{1} a \sec h(\xi)}{b+c \sec h(\xi)}\right] \\
\phi_{5}(x, y, t)= & \left(c_{1}+a_{0}^{2} r_{1}\right) \xi+\frac{a^{2} a_{1}^{2} b r_{1} \sinh (\xi)}{\left(b^{2}-c^{2}\right)(c+b \cosh (\xi))}+\frac{2 a a_{1}\left(a a_{1} c-2 a_{0}\left(b^{2}-c^{2}\right)\right) r_{1}}{\left(b^{2}-c^{2}\right)^{3 / 2}} \\
& \times \tan ^{-1}\left(\frac{(-b+c)}{\sqrt{b^{2}-c^{2}}} \tanh \frac{\xi}{2}\right),
\end{aligned}
$$


where

$$
\begin{gathered}
a_{0}=-\beta c \sqrt{\frac{-1}{2 \lambda\left(c^{2}-b^{2}\right)}}, \quad a_{1}=\frac{\beta}{a} \sqrt{\frac{-2\left(c^{2}-b^{2}\right)}{\lambda}}, \\
\xi=\beta[\mp x+y+(\mp k+c) t], \quad \theta=k x+c y+\left(\frac{1}{2} k^{2}-\frac{1}{2} c^{2} \pm \beta C_{1}\right) .
\end{gathered}
$$

Type 2. If $c_{2}=1, c_{3}=-2 c / a$, and $c_{4}=\left(c^{2}+b^{2}\right) / a^{2}$, then the travelling wave solutions are given as follows:

$$
\begin{aligned}
q_{6}(x, y, t)= & e^{i \theta}\left[a_{0}+\frac{a_{1} a \operatorname{csch}(\xi)}{b+c \csc h(\xi)}\right] \\
\phi_{6}(x, y, t)= & \left(C_{1}+a_{0}^{2} r_{1}\right) \xi-\frac{a^{2} a_{1}^{2} b r_{1} \cosh (\xi)}{\left(b^{2}+c^{2}\right)(c+b \sinh (\xi))}+\frac{2 a a_{1}\left(a a_{1} c+2 a_{0}\left(b^{2}+c^{2}\right)\right) r_{1}}{\left(-b^{2}-c^{2}\right)^{3 / 2}} \\
& \times \tan ^{-1}\left(\frac{(b-c)}{\sqrt{-b^{2}-c^{2}}} \tanh \frac{\xi}{2}\right)
\end{aligned}
$$

where

$$
\begin{gathered}
a_{0}=-\beta c \sqrt{\frac{-1}{2 \lambda\left(c^{2}+b^{2}\right)}}, \\
a_{1}=\frac{\beta}{a} \sqrt{\frac{-2\left(c^{2}+b^{2}\right)}{\lambda}}, \\
\xi=\beta[\mp x+y+(\mp k+c) t], \quad \theta=k x+c y+\left(\frac{1}{2} k^{2}-\frac{1}{2} c^{2} \pm \beta C_{1}\right) .
\end{gathered}
$$

Case 4. If $c_{1}=c_{3}=0$, and $c_{0}, c_{2}, c_{4}$ are arbitrary constants, then $\varphi$ is one of the $16 \varphi_{l}^{I}(l=$ $1,2, \ldots, 16)$ containing $15 \varphi_{l}^{I}$ that are real and one $\varphi_{l}^{I}$ that is complex. For example, if we select $l=4$, then $c_{0}=-1+m^{2}, c_{2}=2-m^{2}$, and $c_{4}=-1$, and the travelling wave solutions are rediscovered:

$$
\begin{gathered}
q_{7}(x, y, t)=a_{1} \operatorname{dn}(\xi) e^{i \theta}, \\
\phi_{7}(x, y, t)=C_{1} \xi+r_{1} a_{0} a_{1}^{2} \int \mathrm{dn}^{2}(\xi) d \xi
\end{gathered}
$$

where

$$
\begin{gathered}
a_{0}=0, \quad a_{1}=\beta \sqrt{\frac{2}{\lambda}}, \quad \lambda>0, \\
\xi=\beta[\mp x+y+(\mp k+c) t], \quad \theta=k x+c y+\left(\frac{1}{2} k^{2}-\frac{1}{2} c^{2} \pm \beta C_{1}\right) .
\end{gathered}
$$


In the limit case, when $m \rightarrow 1$, then (3.20) admits the soliton wave solutions

$$
\begin{aligned}
& q_{7}(x, y, t)=a_{1} \sec h(\xi) e^{i \theta} \\
& \phi_{7}(x, y, t)=C_{1} \xi+r_{1} a_{0} a_{1}^{2} \tanh (\xi)
\end{aligned}
$$

When $m \rightarrow 0$, then (3.20) admits the soliton wave solutions

$$
\begin{gathered}
q_{7}(x, y, t)=a_{1} e^{i \theta}, \\
\phi_{7}(x, y, t)=\left(C_{1}+r_{1} a_{0} a_{1}^{2}\right) \xi
\end{gathered}
$$

where

$$
\begin{gathered}
a_{0}=0, \quad a_{1}=\beta \sqrt{\frac{2}{\lambda}}, \quad \lambda>0 \\
\xi=\beta[\mp x+y+(\mp k+c) t], \\
\theta=k x+c y+\left(\frac{1}{2} k^{2}-\frac{1}{2} c^{2} \pm \beta C_{1}\right) .
\end{gathered}
$$

If we select $l=6$, then $c_{0}=m^{2}, c_{2}=-\left(1+m^{2}\right), c_{4}=1$, and the travelling wave solutions are rediscovered:

$$
\begin{gathered}
q_{7}(x, y, t)=a_{1} \mathrm{~ns}(\xi) e^{i \theta} \\
\phi_{7}(x, y, t)=C_{1} \xi+r_{1} a_{0} a_{1}^{2} \int \mathrm{ns}^{2}(\xi) d \xi
\end{gathered}
$$

where

$$
\begin{gathered}
a_{0}=0, \quad a_{1}=\beta \sqrt{\frac{-2}{\lambda}}, \quad \lambda<0, \\
\xi=\beta[\mp x+y+(\mp k+c) t], \quad \theta=k x+c y+\left(\frac{1}{2} k^{2}-\frac{1}{2} c^{2} \pm \beta C_{1}\right) .
\end{gathered}
$$

In the limit case when $m \rightarrow 1$, then (3.25) admits the soliton wave solutions

$$
\begin{aligned}
& q_{8}(x, y, t)=a_{1} \sec h(\xi) e^{i \theta}, \\
& \phi_{8}(x, y, t)=C_{1} \xi+r_{1} a_{0} a_{1}^{2}(\xi-\operatorname{coth}(\xi)) .
\end{aligned}
$$


When $m \rightarrow 0$, then (3.25) admits the soliton wave solutions

$$
\begin{aligned}
& q_{8}(x, y, t)=a_{1} \csc (\xi) e^{i \theta}, \\
& \phi_{8}(x, y, t)=C_{1} \xi-r_{1} a_{0} a_{1}^{2} \cot (\xi),
\end{aligned}
$$

where

$$
\begin{gathered}
a_{0}=0, \quad a_{1}=\beta \sqrt{\frac{-2}{\lambda}}, \quad \lambda<0, \\
\xi=\beta[\mp x+y+(\mp k+c) t], \quad \theta=k x+c y+\left(\frac{1}{2} k^{2}-\frac{1}{2} c^{2} \pm \beta C_{1}\right) .
\end{gathered}
$$

Case 5. If $c_{2}=c_{4}=0$, then $c_{0}, c_{1}, c_{3}$ are arbitrary constants. The system does not admit solutions of this group.

Case 6. If $c_{0}=c_{1}=c_{2}=c_{3}=0$, and $c_{4}=1$, the travelling wave solutions of (1.1) are

$$
\begin{gathered}
q_{9}(x, y, t)= \pm \frac{a_{1}}{\xi+c} e^{i \theta}, \\
\phi_{9}(x, y, t)=C_{1} \xi-\frac{a_{1}^{2} r_{1}}{\xi+c},
\end{gathered}
$$

where

$$
\begin{gathered}
a_{0}=0, \quad a_{1}=\beta \sqrt{\frac{-2}{\lambda}}, \quad \lambda<0, \\
\xi=\beta[\mp x+y+(\mp k+c) t], \\
\theta=k x+c y+\left(\frac{1}{2} k^{2}-\frac{1}{2} c^{2} \pm \beta C_{1}\right) .
\end{gathered}
$$

Case 7. If $c_{0}=c_{1}=c_{4}=0$, and $c_{2}=1$, then $c_{3}$ is arbitrary constant. The system does not admit solutions of this group.

\section{Summary of the New Compound Riccati Equations Rational Expansion Method}

The key steps of our method are as follows.

Step 1. For a given NLPDEs with some physical fields $u_{i}$ in three variables $x, y, t$,

$$
P_{i}\left(u_{i}, u_{i t}, u_{i x}, u_{i y}, u_{i t t}, u_{i x t}, \ldots\right)=0
$$


by using the wave transformation

$$
\xi=k\left(x+l y-\lambda_{1} t\right), \quad u_{i}(x, y, t)=U(\xi),
$$

where $k, l, \lambda$ are constants to be determined later. Then (4.1) is reduced to an ordinary differential equation

$$
Q\left(U^{\prime}, U^{\prime \prime}, \ldots\right)=0
$$

Step 2. We introduce a solution of (4.3) in terms of finite rational formal expansion in the following forms:

$$
U_{i}(\xi)=a_{0}+\sum_{j=1}^{m i} \frac{\sum_{r_{j_{1}}+r_{j_{2}}=j} a_{r_{j_{1}} r_{j_{2}}}^{i j}\left(\varphi^{\prime \prime}\right)^{r_{j_{1}}}\left(\psi^{\prime}\right)^{r_{j_{2}}}}{\left(\mu_{1} \varphi^{\prime \prime}+\mu_{2} \psi^{\prime}\right)^{j}},
$$

where $a_{r_{1} r_{j_{2}}}^{i j}, \mu_{1}, \mu_{2}\left(r_{j n}=0,1, \ldots, j, n=1,2\right)$ are constants to be determined later and the new variables $\varphi=\varphi(\xi), \psi=\psi(\xi)$ satisfy the Riccati equation.

That is,

$$
\frac{d \varphi}{d \xi}=h_{1}+h_{2} \varphi^{2}, \quad \frac{d \psi}{d \xi}=h_{3}+h_{4} \psi^{2}
$$

where $h_{1}, h_{2}, h_{3}, h_{4}$ are arbitrary constants.

Step 3. The parameter $m_{i}$ can be found by balancing the highest nonlinear terms and the highest-order partial derivative terms in (4.1) or (4.3).

Step 4. Substitute (4.3), (4.4) with (4.5) and then set all coefficients of $\varphi^{i} \psi^{j}[i=1,2, \ldots, j=$ $1,2, \ldots]$ of the resulting system's numerator to zero to get an overdetermined system of nonlinear algebraic system with respect to $\lambda, a_{r_{j_{1}} r_{j_{2}}}^{i j}\left(r_{j n}=0,1, \ldots, j, n=1,2\right)$.

Step 5. Solving the overdetermined system of nonlinear algebraic equations by use of Maple or Mathematica software, we would end up with the explicit expressions for $\lambda, a_{r_{j_{1}} r_{j_{2}}}^{i j}\left(r_{j n}=\right.$ $0,1, \ldots, j, n=1,2)$.

Step 6. It is well known that the general solutions of the Riccati equation

$$
\frac{d F}{d \xi}=r_{1}+r_{2} F^{2}
$$

are the following.

(1) $r_{1}=1 / 2, r_{2}=-1 / 2$,

$$
F(\xi)=\tanh (\xi) \pm i \sec h(\xi), \quad F(\xi)=\operatorname{coth}(\xi) \pm \operatorname{csch}(\xi)
$$


Boundary Value Problems

(2) $r_{1}=r_{2}= \pm 1 / 2$,

$$
F(\xi)=\sec (\xi) \pm i \tan (\xi), \quad F(\xi)=\csc (\xi) \pm \cot (\xi)
$$

(3) $r_{1}=1, r_{2}=-1$,

$$
F(\xi)=\tanh (\xi), \quad F(\xi)=\operatorname{coth}(\xi) .
$$

(4) $r_{1}=r_{2}=1$,

$$
F(\xi)=\tan (\xi)
$$

(5) $r_{1}=r_{2}=-1$,

$$
F(\xi)=\cot (\xi)
$$

(6) $r_{1}=0, r_{2} \neq 0$,

$$
F(\xi)=-\frac{1}{r_{2} \xi+r_{0}}
$$

where $\xi=k\left(x+l y-\lambda_{1} t\right), i=\sqrt{-1}$, and $r_{0}$ is arbitrary constant.

\section{Application of the New Compound Riccati Equations Rational Expansion Method to the Davey-Stewartson (DS) Equations}

By considering the wave transformations,

$$
\begin{gathered}
q(x, y, t)=u(\xi) \exp (i \theta), \quad \phi(x, y, t)=v(\xi) \\
\xi=k\left(x+l y-\lambda_{1} t\right), \quad \theta=k_{1} x+k_{2} y+k_{3} t
\end{gathered}
$$

Equation (1.1) reduces to the following ordinary differential equations:

$$
\begin{gathered}
\sigma^{2} k^{2}\left(1+\sigma^{2}\right) u^{\prime \prime}-2 k u v^{\prime}+2 \lambda u^{3}-\sigma^{2}\left(k_{1}^{2}+\sigma^{2} k_{2}^{2}+2 k_{3}\right) u=0 \\
k\left(1-\sigma^{2} l^{2}\right) v^{\prime \prime}-2 \lambda\left(u^{2}\right)^{\prime}=0
\end{gathered}
$$

where "the prime" denotes to $d / d \xi$. 
Integrating (5.3) w.r.t. $\xi$ and setting the constant of integration to zero, we obtain

$$
k\left(1-\sigma^{2} l^{2}\right) v^{\prime}-2 \lambda u^{2}=0
$$

By balancing the highest nonlinear terms and the highest-Order partial derivative terms in (5.2) and (5.4), we suppose that (5.2) and (5.4) have the following solutions:

$$
\begin{aligned}
& u(\xi)=a_{0}+\frac{a_{1} \varphi^{\prime \prime}+b_{1} \psi^{\prime}}{\mu_{1} \varphi^{\prime \prime}+\mu_{2} \psi^{\prime \prime}} \\
& v(\xi)=b_{0}+\frac{a_{2} \varphi^{\prime \prime}+b_{2} \psi^{\prime}}{\mu_{1} \varphi^{\prime \prime}+\mu_{2} \psi^{\prime \prime}}
\end{aligned}
$$

where $\varphi, \psi$ satisfiy (4.5), with the aid of Mathematica software; substituting (5.5) along with (4.5) into (5.2) and (5.4) yields a set of algebraic equations for $\varphi^{i} \psi^{j}[i, j=0,1,2, \ldots]$ setting the coefficients of these terms $\varphi^{i} \psi^{j}$ to zero yields a set of overdetermined algebraic equations with respect to $a_{0}, a_{1}, b_{1}, b_{0}, a_{2}, b_{2}, l$, and $\lambda_{1}$.

By using the Maple software to solving the overdetermined algebraic equations, we get the following results:

$$
\begin{gathered}
a_{0}=\alpha, \quad a_{1}=\frac{-1}{\alpha \mu_{2}}+1, \\
\quad l= \pm \frac{1}{\sigma}
\end{gathered}
$$

where $\alpha=\left(-\mu_{2} \pm \sqrt{\mu_{2}^{2}+4 \mu_{1} \mu_{2}}\right) / 2 \mu_{1} \mu_{2}$ and $k, \mu_{1}, \mu_{2}, b_{0}, a_{2}, b_{2}, k_{1}, k_{2}, k_{3}$ are arbitrary constants.

So we obtain the following solutions of (1.1).

Family 1. Consider the following

$$
\begin{gathered}
u_{1}=\alpha+\frac{\left(\alpha \mu_{2-1} / \alpha \mu_{2}\right)\left[2 \tanh (\xi)\left(\sec h^{2}(\xi) \mp i \sec h(\xi) \tanh (\xi)\right) \pm i \sec h(\xi)\right]}{\mu_{1}\left[2 \tanh (\xi)\left(\sec h^{2}(\xi) \mp i \sec h(\xi) \tanh (\xi)\right) \pm i \sec h(\xi)\right]+\mu_{2}\left[\csc h^{2}(\xi) \pm \operatorname{csch}(\xi) \operatorname{coth}(\xi)\right]} \\
-\frac{\alpha \mu_{2}\left[\operatorname{csch}^{2}(\xi) \pm \operatorname{csch}(\xi) \operatorname{coth}(\xi)\right]}{\mu_{1}\left[2 \tanh (\xi)\left(\sec h^{2}(\xi) \mp i \sec h(\xi) \tanh (\xi)\right) \pm i \sec h(\xi)\right]+\mu_{2}\left[\csc h^{2}(\xi) \pm \csc h(\xi) \operatorname{coth}(\xi)\right]}, \\
q_{1}(x, y, t)=u_{1} \exp \left[i\left(k_{1} x+k_{2} y+k_{3} t\right)\right] \\
v_{1}=b_{0}+\frac{a_{2}\left[2 \tanh (\xi)\left(\sec h^{2}(\xi) \mp i \sec h(\xi) \tanh (\xi)\right) \pm i \sec h(\xi)\right]+b_{2}\left[\csc h^{2}(\xi) \pm \operatorname{csch}(\xi) \operatorname{coth}(\xi)\right]}{\mu_{1}\left[2 \tanh (\xi)\left(\sec h^{2}(\xi) \mp i \sec h(\xi) \tanh (\xi)\right) \pm i \sec h(\xi)\right]+\mu_{2}\left[\csc h^{2}(\xi) \pm \csc h(\xi) \operatorname{coth}(\xi)\right]}
\end{gathered}
$$


Family 2. Consider the following

$$
\begin{gathered}
u_{2}=\alpha+\frac{\left(\alpha \mu_{2-1} / \alpha \mu_{2}\right)\left[2 \tanh (\xi)\left(\sec h^{2}(\xi) \mp i \sec h(\xi) \tanh (\xi)\right) \pm i \sec h(\xi)\right]}{\mu_{1}\left[2 \tanh (\xi)\left(\sec h^{2}(\xi) \mp i \sec h(\xi) \tanh (\xi)\right) \pm i \sec h(\xi)\right]+\mu_{2}\left[\csc h^{2}(\xi) \pm \csc h(\xi) \operatorname{coth}(\xi)\right]} \\
+\frac{\alpha \mu_{2}\left[\operatorname{csch} h^{2}(\xi) \pm \csc h(\xi) \operatorname{coth}(\xi)\right]}{\mu_{1}\left[2 \tanh (\xi)\left(\sec h^{2}(\xi) \mp i \sec h(\xi) \tanh (\xi)\right) \pm i \sec h(\xi)\right]+\mu_{2}\left[\csc h^{2}(\xi) \pm \csc h(\xi) \operatorname{coth}(\xi)\right]}, \\
q_{2}(x, y, t)=u_{2} \exp \left[i\left(k_{1} x+k_{2} y+k_{3} t\right)\right] \\
v_{2}=b_{0}+\frac{a_{2}\left[2 \tanh (\xi)\left(\sec h^{2}(\xi) \mp i \sec h(\xi) \tanh (\xi)\right) \pm i \sec h(\xi)\right]-b_{2}\left[\csc h^{2}(\xi) \pm \csc h(\xi) \operatorname{coth}(\xi)\right]}{\mu_{1}\left[2 \tanh (\xi)\left(\sec h^{2}(\xi) \mp i \sec h(\xi) \tanh (\xi)\right) \pm i \sec h(\xi)\right]-\mu_{2}\left[\csc h^{2}(\xi) \pm \csc h(\xi) \operatorname{coth}(\xi)\right]}
\end{gathered}
$$

where $\xi=k\left(x \pm(1 / \sigma) y-\sigma^{2}\left(k_{1}+\sigma k_{2}\right) t\right), \alpha=\left(-\mu_{2} \pm \sqrt{\mu_{2}^{2}+4 \mu_{1} \mu_{2}}\right) / 2 \mu_{1} \mu_{2}$ and $k, \mu_{1}, \mu_{2}, b_{0}$, $a_{2}, b_{2}, k_{1}, k_{2}, k_{3}$ are arbitrary constants.

Family 3. Consider the following

$$
\begin{gathered}
u_{3}=\alpha+\frac{\left(\alpha \mu_{2-1} / \alpha \mu_{2}\right)\left[2 \tanh (\xi)\left(\sec h^{2}(\xi) \mp i \sec h(\xi) \tanh (\xi)\right) \pm i \sec h(\xi)\right]}{\mu_{1}\left[2 \tanh (\xi)\left(\sec h^{2}(\xi) \mp i \sec h(\xi) \tanh (\xi)\right) \pm i \sec h(\xi)\right]+\mu_{2}\left[\csc (\xi) \cot (\xi) \pm \csc ^{2}(\xi)\right]} \\
-\frac{\alpha \mu_{2}\left[\csc (\xi) \cot (\xi) \pm \csc ^{2}(\xi)\right]}{\mu_{1}\left[2 \tanh (\xi)\left(\sec h^{2}(\xi) \mp i \sec h(\xi) \tanh (\xi)\right) \pm i \sec h(\xi)\right]+\mu_{2}\left[\csc (\xi) \cot (\xi) \pm \csc ^{2}(\xi)\right]} \\
q_{3}(x, y, t)=u_{3} \exp \left[i\left(k_{1} x+k_{2} y+k_{3} t\right)\right] \\
v_{3}=b_{0}+\frac{a_{2}\left[2 \tanh (\xi)\left(\sec h^{2}(\xi) \mp i \sec h(\xi) \tanh (\xi)\right) \pm i \sec h(\xi)\right]+b_{2}\left[\csc (\xi) \cot (\xi) \pm \csc ^{2}(\xi)\right]}{\mu_{1}\left[2 \tanh (\xi)\left(\sec h^{2}(\xi) \mp i \sec h(\xi) \tanh (\xi)\right) \pm i \sec h(\xi)\right]+\mu_{2}\left[\csc (\xi) \cot (\xi) \pm \csc ^{2}(\xi)\right]}
\end{gathered}
$$

Family 4. Consider the following

$$
\begin{gathered}
u_{4}=\alpha+\frac{\left(\alpha \mu_{2-1} / \alpha \mu_{2}\right)\left[2 \operatorname{coth}(\xi)\left(\csc h^{2}(\xi) \pm \cot h(\xi) \csc h(\xi)\right) \mp \csc h(\xi)\right]}{\mu_{1}\left[2 \operatorname{coth}(\xi)\left(\csc h^{2}(\xi) \pm \cot h(\xi) \csc h(\xi)\right) \pm i \sec h(\xi)\right]-\mu_{2}\left[\csc h^{2}(\xi) \pm \csc h(\xi) \operatorname{coth}(\xi)\right]} \\
+\frac{\alpha \mu_{2}\left[\csc h^{2}(\xi) \pm \csc h(\xi) \operatorname{coth}(\xi)\right]}{\mu_{1}\left[2 \operatorname{coth}(\xi)\left(\csc h^{2}(\xi) \pm \cot h(\xi) \csc h(\xi)\right) \pm i \sec h(\xi)\right]-\mu_{2}\left[\csc h^{2}(\xi) \pm \csc h(\xi) \operatorname{coth}(\xi)\right]}, \\
q_{4}(x, y, t)=u_{4} \exp \left[i\left(k_{1} x+k_{2} y+k_{3} t\right)\right], \\
v_{4}=b_{0}+\frac{a_{2}\left[2 \operatorname{coth}(\xi)\left(\csc h^{2}(\xi) \pm \cot h(\xi) \csc h(\xi)\right) \mp \csc h(\xi)\right]-b_{2}\left[\csc h^{2}(\xi) \pm \csc h(\xi) \operatorname{coth}(\xi)\right]}{\mu_{1}\left[2 \operatorname{coth}(\xi)\left(\csc h^{2}(\xi) \pm \cot h(\xi) \csc h(\xi)\right) \pm i \sec h(\xi)\right]-\mu_{2}\left[\csc h^{2}(\xi) \pm \csc h(\xi) \operatorname{coth}(\xi)\right]} .
\end{gathered}
$$


Family 5. Consider the following

$$
\begin{gathered}
u_{5}=\alpha+\frac{\left(\alpha \mu_{2-1} / \alpha \mu_{2}\right)\left[2 \operatorname{coth}(\xi)\left(\csc h^{2}(\xi) \pm \cot h(\xi) \csc h(\xi)\right) \mp \csc h(\xi)\right]}{\mu_{1}\left[2 \operatorname{coth}(\xi)\left(\csc h^{2}(\xi) \pm \cot h(\xi) \csc h(\xi)\right) \pm i \sec h(\xi)\right]+\mu_{2}\left[\sec (\xi) \tan (\xi) \pm \sec ^{2}(\xi)\right]} \\
-\frac{\alpha \mu_{2}\left[\sec (\xi) \tan (\xi) \pm \sec ^{2}(\xi)\right]}{\mu_{1}\left[2 \operatorname{coth}(\xi)\left(\csc h^{2}(\xi) \pm \cot h(\xi) \csc h(\xi)\right) \pm i \sec h(\xi)\right]+\mu_{2}\left[\sec (\xi) \tan (\xi) \pm \sec ^{2}(\xi)\right]} \\
q_{5}(x, y, t)=u_{5} \exp \left[i\left(k_{1} x+k_{2} y+k_{3} t\right)\right] \\
v_{5}=b_{0}+\frac{a_{2}\left[2 \operatorname{coth}(\xi)\left(\csc h^{2}(\xi) \pm \cot h(\xi) \csc h(\xi)\right) \mp \csc h(\xi)\right]+b_{2}\left[\sec (\xi) \tan (\xi) \pm \sec ^{2}(\xi)\right]}{\mu_{1}\left[2 \operatorname{coth}(\xi)\left(\csc h^{2}(\xi) \pm \cot h(\xi) \csc h(\xi)\right) \pm i \sec h(\xi)\right]+\mu_{2}\left[\csc h^{2}(\xi) \pm \csc h(\xi) \operatorname{coth}(\xi)\right]}
\end{gathered}
$$

Family 6. Consider the following

$$
\begin{aligned}
u_{6}= & \alpha+\frac{\left(\alpha \mu_{2-1} / \alpha \mu_{2}\right)\left[2 \operatorname{coth}(\xi)\left(\csc h^{2}(\xi) \pm \cot h(\xi) \csc h(\xi)\right) \mp \csc h(\xi)\right]}{\mu_{1}\left[2 \operatorname{coth}(\xi)\left(\csc h^{2}(\xi) \pm \cot h(\xi) \csc h(\xi)\right) \pm i \sec h(\xi)\right]-\mu_{2}\left[\csc (\xi) \cot (\xi) \pm \csc ^{2}(\xi)\right]} \\
& +\frac{\alpha \mu_{2}\left[\csc (\xi) \cot (\xi) \pm \csc ^{2}(\xi)\right]}{\mu_{1}\left[2 \operatorname{coth}(\xi)\left(\csc h^{2}(\xi) \pm \cot h(\xi) \csc h(\xi)\right) \pm i \sec h(\xi)\right]-\mu_{2}\left[\csc (\xi) \cot (\xi) \pm \csc ^{2}(\xi)\right]} \\
q_{6}(x, y, t)=u_{6} \exp \left[i\left(k_{1} x+k_{2} y+k_{3} t\right)\right] & \\
v_{6}= & b_{0}+\frac{a_{2}\left[2 \operatorname{coth}(\xi)\left(\operatorname{csch}^{2}(\xi) \pm \cot h(\xi) \csc h(\xi)\right) \mp \csc h(\xi)\right]-b_{2}\left[\csc (\xi) \cot (\xi) \pm \csc ^{2}(\xi)\right]}{\mu_{1}\left[2 \operatorname{coth}(\xi)\left(\csc h^{2}(\xi) \pm \cot h(\xi) \csc h(\xi)\right) \pm i \sec h(\xi)\right]-\mu_{2}\left[\csc (\xi) \cot (\xi) \pm \csc ^{2}(\xi)\right]}
\end{aligned}
$$

where $\xi=k\left(x \pm(1 / \sigma) y-\sigma^{2}\left(k_{1}+\sigma k_{2}\right) t\right), \alpha=\left(-\mu_{2} \pm \sqrt{\mu_{2}^{2}+4 \mu_{1} \mu_{2}}\right) / 2 \mu_{1} \mu_{2}$, and $k, \mu_{1}, \mu_{2}, b_{0}, a_{2}, b_{2}, k_{1}, k_{2}, k_{3}$ are arbitrary constants.

\section{Conclusion}

In this paper, we have used the extended Fan's subequation method and a new compound Riccati equations rational expansion method to construct the exact travelling wave solutions and obtain many explicit solutions for the Davey-Stewartson equations.

We deduced a relation between the general elliptic equation involving five parameters and other subequations involving three parameter, like Riccati equation, auxiliary ordinary equation, first-kind elliptic equation, and generalized Riccati equation; many exact travelling wave solutions and new complexion solutions including more general soliton solutions, triangular solutions, double-periodic solutions, hyperbolic function solutions, and trigonometric function solutions are also given. 


\section{References}

[1] E. G. Fan, "Darboux transformation and soliton-like solutions for the Gerdjikov-Ivanov equation," Journal of Physics A, vol. 33, no. 39, pp. 6925-6933, 2000.

[2] H.-W. Tam, W.-X. Ma, X.-B. Hu, and D.-L. Wang, "The Hirota-Satsuma coupled KdV equation and a coupled Ito system revisited," Journal of the Physical Society of Japan, vol. 69, no. 1, pp. 45-52, 2000.

[3] E. M. E. Zayed, H. A. Zedan, and K. A. Gepreel, "Group analysis and modified extended tanhfunction to find the invariant solutions and soliton solutions for nonlinear Euler equations," International Journal of Nonlinear Sciences and Numerical Simulation, vol. 5, pp. 221-234, 2004.

[4] Y. Lei, Z. Fajiang, and W. Yinghai, "The homogeneous balance method, Lax pair, Hirota transformation and a general fifth-order KdV equation," Chaos, Solitons and Fractals, vol. 13, no. 2, pp. 337-340, 2002.

[5] M. McConnell, A. S. Fokas, and B. Pelloni, "Localised coherent solutions of the DSI and DSII equations-a numerical study," Mathematics and Computers in Simulation, vol. 69, no. 5-6, pp. 424-438, 2005.

[6] A. Davey and K. Stewartson, "On three-dimensional packets of surface waves," Proceedings of the Royal Society A, vol. 338, pp. 101-110, 1974.

[7] Sirendaoreji and S. Jiong, "Auxiliary equation method for solving nonlinear partial differential equations," Physics Letters A, vol. 309, no. 5-6, pp. 387-396, 2003.

[8] F. Xie, Y. Zhang, and Z. Lü, "Symbolic computation in non-linear evolution equation: application to $(3+1)$-dimensional Kadomtsev-Petviashvili equation," Chaos, Solitons and Fractals, vol. 24, no. 1, pp. 257-263, 2005.

[9] E. G. Fan, "Uniformly constructing a series of explicit exact solutions to nonlinear equations in mathematical physics," Chaos, Solitons and Fractals, vol. 16, no. 5, pp. 819-839, 2003.

[10] E. Yomba, "The extended Fan's sub-equation method and its application to KdV-MKdV, BKK and variant Boussinesq equations," Physics Letters A, vol. 336, no. 6, pp. 463-476, 2005.

[11] A. A. Soliman and M. A. Abdou, "Exact travelling wave solutions of nonlinear partial differential equations," Chaos, Solitons and Fractals, vol. 32, no. 2, pp. 808-815, 2007.

[12] L.-N. Song, Q. Wang, and H.-Q. Zhang, "A new compound Riccati equations rational expansion method and its application to the (2+1)-dimensional asymmetric Nizhnik-Novikov-Vesselov system," Chaos, Solitons and Fractals, vol. 36, no. 5, pp. 1348-1356, 2008. 